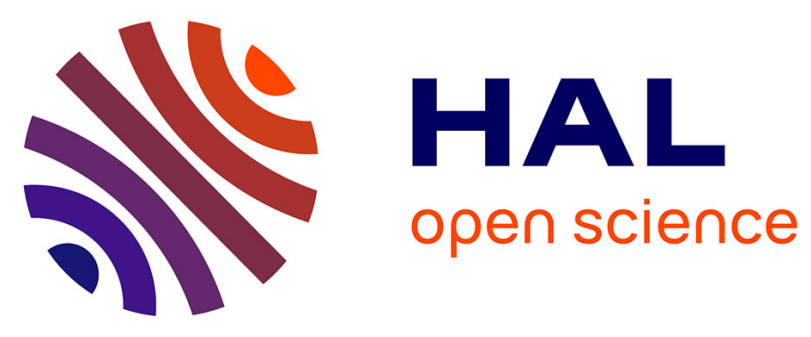

\title{
Energy-Based Functional Modelling for Control Architecture Design: An Application to Energy Management for a Hybrid Electric Vehicle
}

Mert Mokukcu, Philippe Fiani, Sylvain Chavanne, Lahsen Ait Taleb, Cristina Vlad, Emmanuel Godoy

\section{To cite this version:}

Mert Mokukcu, Philippe Fiani, Sylvain Chavanne, Lahsen Ait Taleb, Cristina Vlad, et al.. EnergyBased Functional Modelling for Control Architecture Design: An Application to Energy Management for a Hybrid Electric Vehicle. Oleg Gusikhin; Kurosh Madani. Informatics in Control, Automation and Robotics. 14th International Conference, ICINCO 2017 Madrid, Spain, July 26-28, 2017 Revised Selected Papers, 495, Springer, pp.47-72, 2020, Lecture Notes in Electrical Engineering, 10.1007/9783-030-11292-9_3. hal-02110024

\section{HAL Id: hal-02110024 \\ https://hal.science/hal-02110024}

Submitted on 16 Mar 2020

HAL is a multi-disciplinary open access archive for the deposit and dissemination of scientific research documents, whether they are published or not. The documents may come from teaching and research institutions in France or abroad, or from public or private research centers.
L'archive ouverte pluridisciplinaire HAL, est destinée au dépôt et à la diffusion de documents scientifiques de niveau recherche, publiés ou non, émanant des établissements d'enseignement et de recherche français ou étrangers, des laboratoires publics ou privés. 


\title{
Energy-based Functional Modelling for Control Architecture Design: an Application to Energy Management for a Hybrid Electric Vehicle.
}

\author{
Mert Mökükcü ${ }^{1,2[0000-0003-0848-2983]}$, Philippe Fiani ${ }^{1[0000-0002-6215-5601]}$, Sylvain Chavanne ${ }^{1}$, \\ Lahsen Ait Taleb ${ }^{1}$, Cristina Vlad ${ }^{1[0000-0003-0250-5785]}$ and Emmanuel Godoy ${ }^{2[0000-0001-9114-}$ \\ 8729] \\ ${ }^{1}$ Sherpa Engineering, R\&D Department, 92250, La Garenne Colombes, France \\ m.mokukcu,p.fiani, s. chavanne, l.aittaleb@sherpa-eng.com \\ ${ }^{2}$ Laboratoire des Signaux et Systèmes (L2S, UMR CNRS 8506), Centrale Supélec - CNRS - \\ Univ. Paris-Sud, Univ. Paris Saclay, 3 rue Joliot Curie, F-91192, Gif-sur-Yvette cedex, France- \\ cristina.vlad, emmanuel.godoy@centralesupelec.fr
}

\begin{abstract}
The increasing level of complexity of energy systems drives researchers to focus their studies on energy optimization, by using modelling and simulation methods capable to represent the real system behavior. In this study, a functional energetic modeling method is used to design a control architecture for energy flow management, which relies on local control loops, a decision manager (DM) and basic equations. When the functional level of representation is used to model a complex system, the evaluation of model accuracy (from an energetic point of view) and the validation of energy management algorithms are eased by fast simulations due to low model complexity. While the functional model allows a first-stage validation of energy distribution within the system, the energy management algorithms need to be tested using a more accurate model, which is the multi-physical model of the system. The multi-physical model has its own local controllers and a global resource manager (GRM) to handle the power split between different components. The second-stage validation can be completed by adapting the functional model in order to design the high-level controller, the GRM, at multi-physical level. To develop the control architecture of the multi-physical model based on the functional model, two steps are required: i) adjust the parametrization of functional elements and $\mathrm{ii}$ ) propose a method to interconnect the models at both levels of representation (functional/ multiphysical level). Thus, an example of a hybrid electric vehicle (HEV) is considered for functional elements modelling and parametrization. In addition, the GRM design is presented and simulation results of the HEV system at multi-physical level are illustrated to validate the system architecture and component sizing, and to evaluate the fuel consumption compared with $\mathrm{HEV}$ design specifications.
\end{abstract}

Keywords: control design, energy management, complex systems, modeling, integrated design, systems modeling, interconnected systems, system-level design, system verification, automotive engineering, system architecture. 


\section{Introduction}

It is important to cite economic and ecological framework that drags industry and research towards an innovative energy management involving an association of energy technologies, optimal control laws and refined components. Technological advancements on components technology, component-to-component communication and data acquisition methods make systems more intelligent, but also more complex. The high complexity of energy systems renders the control design and energy optimization more difficult, which enforces the research on system engineering. The most important challenges on this topic can be summarized as follows:

- Choosing the system architecture;

- Setting and sizing the system components;

- Optimizing the flow between multi-sources and multi-consumers;

- Designing a control system architecture.

To handle these challenges, research focused on developing modeling formalisms and simulation tools that allow obtaining the following results:

- A global and interactive approach to improve systematic innovation;

- A methodology for architecture evaluation and system verification from the early stages of the system life cycle;

- A system representation from multiple points of view to define and analyze the main objectives;

- Definition of a control system.

A solution to improve the time to market is to represent a complex system at a higher level of abstraction, which will ease its global understanding within a structured environment. In the literature, this type of representation is associated with systemic theory [5]. Usually, physical models of complex systems have been represented and analyzed using Bond Graph modeling and multi-domain simulation [1]. However, another interesting approach is the functional modeling $[6,7,10]$ that is based on the following principle: a system can be defined by basic elements, modelled with an adequate level of complexity, which faithfully describe the system behavior.

By construction, Model Based System Engineering (MBSE) allows to specify and design systems at different levels of abstraction and to specify their elements and the links between them. These links are: components and information, requirements, architecture (functional, multi-physical or otherwise), use cases and validation tests [4].

In [4] and [6], three levels of modeling are introduced as:

1. Teleological modeling: define a system of missions (set of services to be realized by the system) and the purpose of the system in its environment.

2. Functional modeling: define the main functions of the system of missions and the associated architecture.

3. Multi-physical modeling: model the components of the system and the equipment provided by suppliers. 


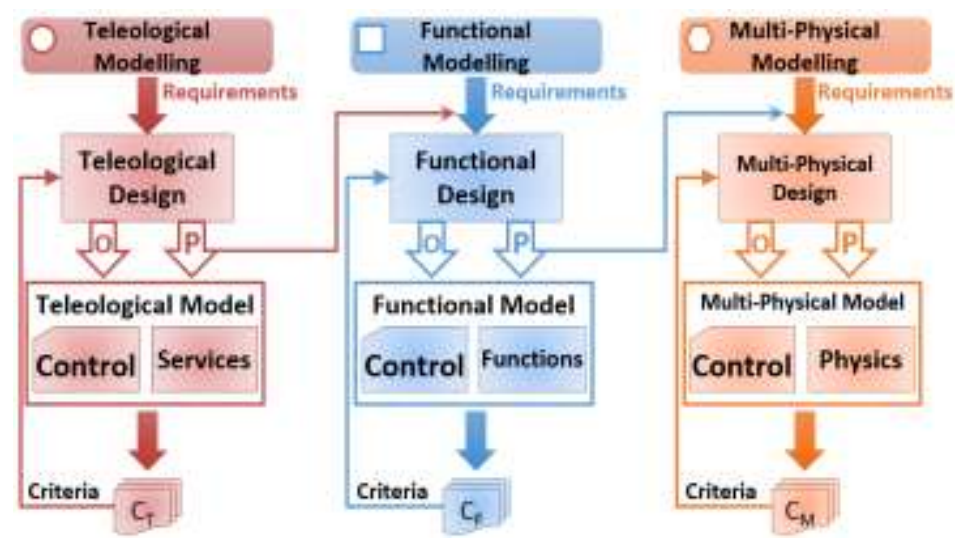

Fig. 1. Multi-level integrated design and simulation [4].

Fig. 1 illustrates the modeling steps for each system representation at a different level of abstraction. The development starts with requirements formulation. Once the requirements are fixed, the parameters $(\mathrm{P})$ and objectives $(\mathrm{O})$ are defined in order to obtain a simulation model and its associated controller. The resulting control system is evaluated in simulation using validation criteria. If the criteria are satisfied then the parameters of higher levels of modeling will define the requirements of lower levels of modeling. Otherwise, necessary modifications are made in the design process. This mechanism helps to pass on objectives or parameters between different representations of the system. Besides, it can allow a higher modeling level to become the controller of a lower modeling level [4].

At functional level of abstraction, the system behavior is represented from an energetic point of view, using simple equations to reduce the amount of time needed to complete a simulation. The functional modeling methodology and its semantics [2,3, 6] are based on FUs (Functional Units), also referred as OFS (Organico-Functional Sets). In Fig. 2, the representation of a functional model is given. Each element " $\Sigma$ " represents a functional unit while D elements are used for energy distribution [6].

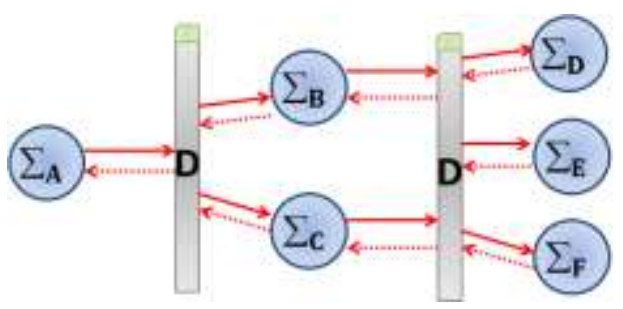

Fig. 2. Functional modeling representation [11].

However, at multi-physical level, the system can be represented as a composition of controlled subsystems [6]. The block diagram of a multi-physical representation is shown in Fig. 3, where $C, I, T$ and $E$ denote the local controller, input conditioning, 
transformer and effector, respectively. The Global Resource Manager (GRM) block acts like an energy management system for the multi-physical model.

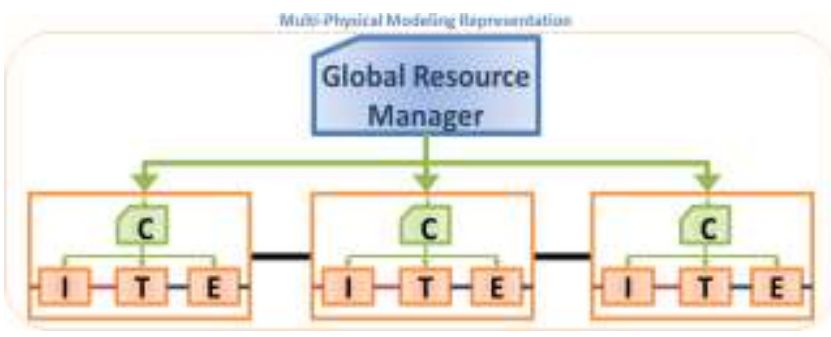

Fig. 3. Multi-physical modeling representation [11].

Indeed, in the early stages of the design process, a functional model is preferred to represent the complex system in order to validate (by fast simulations) the system architecture and components sizing, and also to evaluate the performances of local controllers and energy management strategies for different missions using different criteria. Naturally, the next stage in the system design process is to test the supervision and control algorithms that have been developed using the functional model, on the multiphysical model. In Fig. 4, the control architecture is illustrated at functional and multiphysical levels of abstraction. On the left side of the figure, the system is represented at functional level, where the energy flow within the system is managed by the supervision block DM (Decision Manager) using optimization algorithms. On the right side, $C$ is a composition of local controllers of the system, and $P$ includes physical subsystems. Therefore, the main difficulty is to obtain the global resource manager (GRM) of the multi-physical model using information provided by local controllers and decision manager (DM) of the functional model.

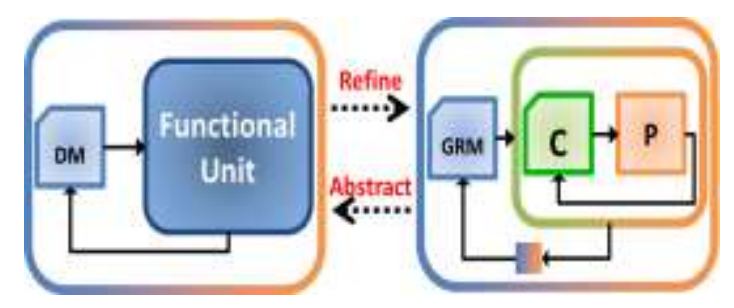

Fig. 4. Control system representation [11].

The problem can be formulated in the following manner: Which input/output of which physical subsystem should be measured/estimated? How to use these signals to provide energy transfer information to functional model? After processing this information, how to transform the computed power reference into a physical reference signal and transfer it to different types of controllers of physical subsystems?

For a better understanding of these challenges in the context of an energy system, a hybrid electric vehicle (HEV) is considered as an example of a multi-source/multi-consumer system. 
In this work, the issue of interconnecting the functional and multi-physical models is presented, and a solution is proposed showing how the GRM can be extracted from the functional representation and be connected to local controllers of the multi-physical model. In Section 2, the multi-physical and functional modeling methods are briefly introduced. In addition, it is described how the functional model parametrization can be translated into a parametrization of a group of components at multi-physical level. In Section 3, the interconnection procedure between the two modeling levels is discussed. Section 4 presents both models for a plug-in parallel hybrid vehicle, along with the multi-physical model obtained as a result of interconnection. Its performances are tested in simulation for a specific mission. Conclusions and future work are summarized in Section 5.

\section{Modeling Method Reminders}

This section introduces briefly the multi-physical and functional modeling methods, which are further applied to model the behavior of a gear motor group at multi-physical and functional level, respectively.

\subsection{Multi-Physical Modeling}

Multi-physical modeling aims to represent the technological equipment architecture of a complex system. Generally, the 0D-1D multi-physical modeling is used in an industrial environment for sizing optimization, control laws design and validation. This multi-physical model allows representing the complex system as a whole and is used for simulations, analysis and prediction of system performances.

The multi-physical model is composed by analytical models that provide an accurate description of the multi-physical behavior of the complex system. The multi-physical model can be developed under the simulation environment, for example Matlab/Simulink, using a component-based approach derived from the Bond Graph methodology. In Bond Graph language, the passage between physical and mathematical models is done using a block-diagram environment [1]. On the other hand, the simulation tool is based on a multi-port concept: a unique link is used to represent and simulate all the interactions between different components. In the multi-physical methodology, this link is represented by energy transfer. Moreover, every link between physical model components consists of a flux variable and an effort variable that depend on the physical domain. In Fig. 5, some examples are given for different domains. Despite the advantages of multi-physical modeling (accuracy and intermediate signals availability), the model design, its simulation and validation are time consuming and require expertise. 


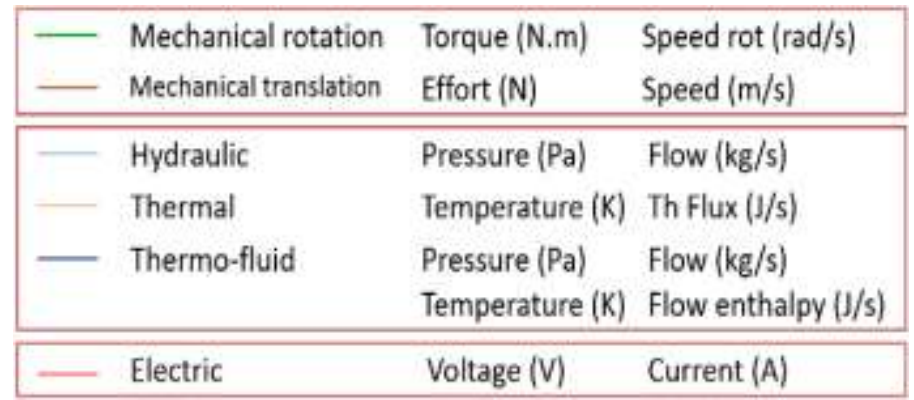

Fig. 5. Multi-physical domains [11].

For these reasons, it is necessary to use a model of a higher level of abstraction, which does not need the definition of multi-physical elements, in order to easily evaluate the system in the early stages of the design process.

An example of multi-physical model for a gear motor group is given in Fig. 6(a). The transformation of electrical flow into mechanical flow is done using a converter (1), an electrical motor (2), (3) and a gear reducer (4), along with their local controller. For this example, the physical behavior of each component is represented by a simple analytical model as follows:

$$
\begin{gathered}
u_{R}=u_{E} \cdot \frac{t_{\text {on }}}{t_{\text {on }}+t_{\text {off }}} \\
u_{R}=R_{R} \cdot i_{R}+L_{R} \cdot \frac{d i_{R}}{d t}+E \\
J_{C R} \cdot \frac{d^{2} \theta_{m}}{d t^{2}}=\tau_{\text {em }}-\tau_{p}-\tau_{\text {ext }} \\
\tau_{\text {ext }}=\alpha \cdot \tau_{\text {out }}
\end{gathered}
$$

where $u_{R}$ denotes the rotor voltage; $u_{E}$ is the converter supply voltage; $t_{\text {on }}$ and $t_{\text {off }}$ are the converter on and off time, respectively; $R_{R}, i_{R}, L_{R}$ is the resistance, current and inductance of the rotor, respectively; $E$ is the electromotive force; $J_{C R}$ is the inertia; $\theta_{m}$ is the motor angular position; $\tau_{e m}$ is the electromagnetic torque, $\tau_{p}$ is the loss torque; $\tau_{\text {ext }}$ is the motor output torque; $\alpha$ is the gear constant and $\tau_{\text {out }}$ is the gear output torque.

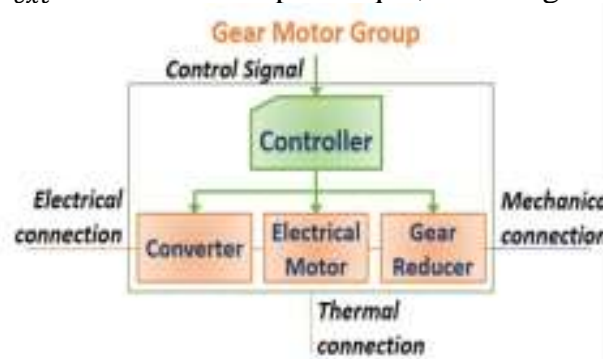

(a)

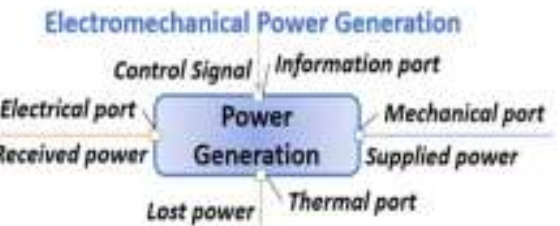

(b)

Fig. 6. Multi-physical (a) \& functional (b) model representations [11]. 


\subsection{Functional Modeling}

The concept of functional modeling has been introduced and detailed in [6]. Unlike multi-physical modeling concept, in this methodology, functional links are employed to represent flow exchanges within the complex system. In a functional model, the flow can be either energy, either matter or both, and it is exchanged together with an information flow. The exchange of flow is made between five types of elements (source, storage, distribution, transmission and effector), each of them having source and consumer ports. Source ports receive expressed need from consumer ports and they answer by supplying the requested need. In addition, consumer ports transmit demands of need to source ports. This gives the method its modularity. Brief information about basic elements of functional modeling is given in Table.1. The functional model can be simulated using, for example, the simulation environment Matlab/Simulink, with a functional modeling library, which contains all these basic elements.

Table 1. Element types of functional energetic modeling and associated functions [6].

\begin{tabular}{|c|c|c|c|c|}
\hline Source & Storage & Transformation & Distribution & Effector \\
\hline Energy \& & $\begin{array}{c}\text { Energy \& } \\
\text { Matter } \\
\text { Storage }\end{array}$ & $\begin{array}{c}\text { Energy \& Matter } \\
\text { Transformation in } \\
\text { Different Domains }\end{array}$ & $\begin{array}{c}\text { Energy \& } \\
\text { Matter Dis- } \\
\text { tribution }\end{array}$ & $\begin{array}{c}\text { Energetic } \\
\text { Services }\end{array}$ \\
\hline
\end{tabular}

In a functional model, the need computation starts from the effector. For example, the need of energy for a plug-in hybrid electric vehicle (PHEV) is calculated by electrical auxiliary element or vehicle dynamics element (both of them effectors of the system). Then, the energy need is sent to storages or sources via distribution and transformation elements. Based on information flow, the storages and sources can decide whether they are able to provide the requested energy or not. Furthermore, distribution elements are used to manage the energy flow between sources and storages, and to supply the requested energy to effectors as an answer to their need. If a hotel water treatment system is considered, the need of water consumption is calculated by hotel consumer element (effector) and the hotel logistics element must supply the required amount of water, with suitable properties.

Fig. 6(b) illustrates the functional model of a gear motor group, which also represents an energy transformation element (transformation of electrical energy into mechanical and thermal energy) but without considering the real physical behavior. The model is described as below:

$$
P_{\text {mech }}=\eta \cdot P_{e l} \text { Fig }
$$

where $P_{\text {mech }}$ denotes the mechanical output power, $P_{e l}$ is the electrical input power and $\eta$ is the efficiency. Besides (5), maximum and minimum power limitations, $P_{\max }$ and $P_{\text {min }}$, are specified for the transformation element.

Moreover, functional elements can be represented by static or dynamic models. The dynamic behavior is taken into account either by an integration for the energy-to-power transition, either by adding $1^{\text {st }}$ (or $2^{\text {nd }}$ ) order transfer functions of different elements 
such as transformations, storages or effectors. Using the functional modelling formalism, the energetic model allows to perform fast simulations for system evaluation (sizing, architecture, requirements management) before choosing the technology, to obtain the GRM for the multi-physical model and to simulate the system (multi-physical model having the functional model as energy management system) as a whole.

The next sections describe how the functional model parametrization is derived from the parametrization of a group of components at multi-physical level. Then, the solution used to interconnect the functional and multi-physical models is presented, which comes to design the GRM based on the functional model.

\subsection{Functional Model Design and Parametrization based on Multi-Physical Component Groups}

Besides a fast evaluation of system performances from an energetic point of view, the functional model is essential for the control architecture design at multi-physical level of abstraction.

As each functional unit of the functional model represents one or multiple components of multi-physical model, the functional model design based on an existing multiphysical model plays a major role in accelerating the system design and validation process. However, setting the functional model parameters in the early stages of system design can be a challenging task. If the functional model is used to model the control architecture at multi-physical level, it can be used as an energy management control system. In this case, the parameters of the functional model need to be adapted so that they can be transferred between multi-physical level and functional level. Nonetheless, in the design process, before using this model to build a control architecture, the energy management strategy (i.e. optimization algorithms based on priorities) is validated at functional level. In addition, this model is used to check the parameters sizing of functional units (functional elements representing functions of component groups with local controllers at multi-physical level).

If the functional elements sizing and the distribution priorities are established based on a poor parametrization, the optimality of resource allocation and distribution is lost and the functional model cannot serve to design the control architecture of the multiphysical model. Improper parametrization can lead to serious complications at functional and multi-physical level such as exceeding power limitations, wrong resource allocation, wrong measurements that can make local control-loops instable. This is why it is necessary to adapt the functional model elements to the multi-physical model.

An example of functional element design and parametrization is illustrated in Section 4.

\section{Interconnection Between Functional and Multi-Physical Modeling}

Functional modeling defines key-functions (FUs), allocates and refines end-mission requirements to the FUs and defines the energy management system. On the other hand, 
multi-physical modeling defines the physical architecture or physical units, allocates and refines the functional requirements to physical units. To overcome the challenges associated with the control laws design and energy management within the entire system, functional and multi-physical models are interconnected. In this section, the problems related to the interconnection are presented along with the proposed solution. This solution is presented for the gear motor group and the electromechanical energy transformation that are introduced as examples in Section 2.

\subsection{Problems of Interconnection}

As presented in Fig. 4, the functional modeling level includes a control strategy that will be used by the control system of the multi-physical model. Moreover, this strategy is independent from technical components, and is defined according to the decision manager allocated from end-missions model.

In Fig. 7, the representations of control systems and flow exchanges of functional and multi-physical models are shown for a battery electric vehicle (BEV). Here, the challenge is to find the adequate language to connect both models.

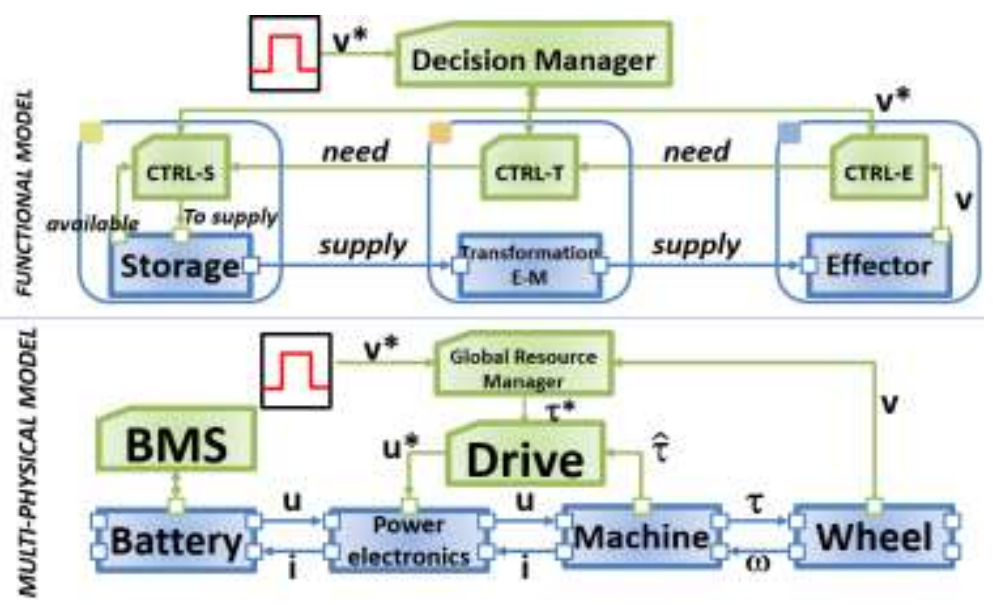

Fig. 7. Control system and flow exchanges of functional and multi-physical models [11].

As flux exchanges are different between functional and multi-physical models, the connection cannot be done directly. Since multi-physical model components need physical domain references and functional model components require a power demand reference for simulation, connecting the power flow to physical domain flows can be a challenging task. At this stage, the interesting features of the interconnection can be expressed as follows:

- Functional modeling allows fast control architecture design and fast adaptation to eventual changes in the system, 
- Multi-physical representation is too complex and time consuming when trials are accomplished.

\subsection{Proposed Solution}

A solution to the interconnection problem is to build an interface between the multiphysical and functional model. As illustrated in Fig. 8, this interface contains passage equations between physical domain and functional domain. It accomplishes the following functions: determine the equivalent physical references required for the multi-physical model based on the power demand provided by the functional model; measure/estimate the power supply that the system is able to deliver using information from the multi-physical model, and transfer the estimated power supply to the functional model. For each functional model element, an interface is required in order to calculate/adapt the necessary values.

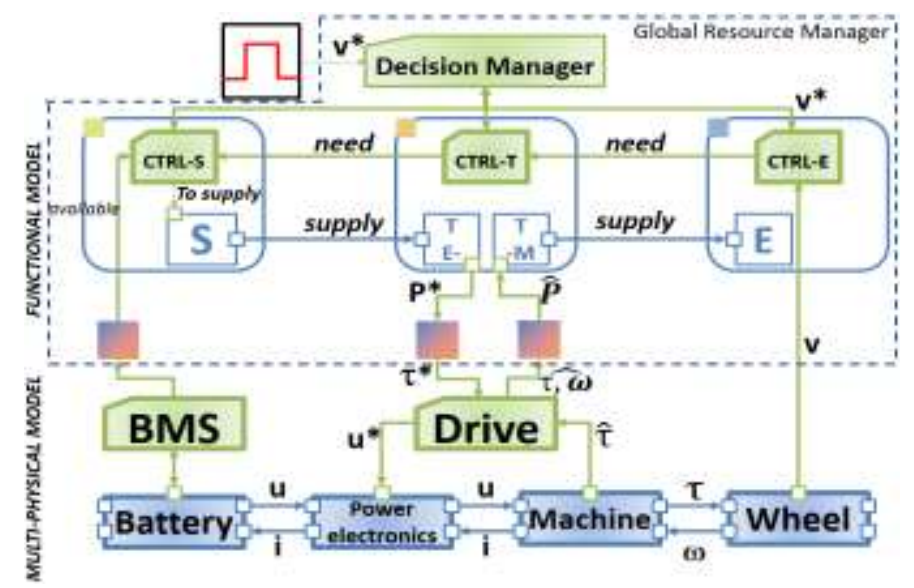

Fig. 8. Functional to multi-physical domain interconnection [11]

If the electromechanical transformation element is considered, the interface between this element and electrical propulsion group (drive and electric machine in this example) uses the following equations:

$$
\begin{gathered}
\frac{P_{f n c}^{*}}{\left|\widehat{w_{r}}\right|}=\tau_{c n s}^{*} \\
P_{\text {mech }}=\hat{P}_{f n c}
\end{gathered}
$$

where $P_{f n c}^{*}$ denotes the power demand; $\widehat{w_{r}}$ is estimated/ measured angular speed of rotor; $\tau_{c n s}^{*}$ is torque demand; $P_{\text {mech }}$ is calculated mechanical power of the electrical machine and $\hat{P}_{f n c}$ is estimated/measured output power of the motor.

In the next section, an example of PHEV is presented. First of all, model architectures of functional and multi-physical models are given, and secondly, simulation results using the functional model and the multi-physical model with GRM are discussed. 


\section{Application to a Hybrid Vehicle Energy Management System}

\subsection{Motive}

Despite their high performances, economic advantages and maintenance costs, electrified vehicles have been abandoned until last decade. The increasing pollution caused by conventional vehicles, the decrease in oil reserves and the rising fuel prices triggered the need for fuel economy and motivated the research on electrified vehicles, especially on fuel cell and hybrid electric vehicles. Considering that the fuel cell vehicles are currently in development process, hybrid electric vehicles (HEVs) are assumed as the most viable solution for the coming years [8]. As the research on HEVs grows bigger, component technology is also advancing. Along with this technology advancement, the system becomes more complex to control which makes energy management and control strategies for HEVs to be an important research field.

In an HEV, and internal combustion engine (ICE) and one or several additional electric motors (EMs) are used for the vehicle powertrain. The ICE is supplied by fuel while the EMs are supplied by batteries. These components, usually allowing different possible interconnections, form a complex and challenging multi-source/multi-consumer system in terms of optimal control design and energy management. Both objectives of the design process have to satisfy several vehicle services like fuel consumption or comfort level. Although there are optimization methods applied on HEVs, they are implemented for a specific architecture of the HEV and they usually require a priori knowledge of the driving cycle. Thus, the question is how to manage the power split that globally satisfies the vehicle services whenever the vehicle has a new task [8] and/or the system architecture is reconfigured.

In this study, a parallel plug-in hybrid electric vehicle (PHEV) is considered due to the resemblance to a battery electric vehicle that has been highly investigated over the last few years.

\subsection{Functional Model Design and Parametrization for PHEV}

The first step in the design of a functional model is to define key functions of the system without ignoring that every system has at least one source and one effector element. If an HEV is considered, the sources are the fuel station and electrical grid, and the effectors are the vehicle services such as mobility (energy need calculated by vehicle dynamics) and thermal comfort (energy need calculated by thermal balance equations). As for the energy storages of the functional model, the battery and the converters form the electrical storage element and the fuel tank with its pumping system form the fuel storage element. Transformation elements are defined by regrouping traction components, since their functionality is transforming the physical domain. There are three transformation elements for an HEV system: F2M (fuel to mechanical) energy transformation, M2E (mechanical to electrical) energy transformation and E2M (electrical to mechanical) energy transformation that represent the energetic functions of the following groups of multi-physical components: \{internal combustion engine (ICE) + gearbox $\}$, \{electric generator $\}$ and $\{$ inverter + electric motor + rectifier $\}$, respectively. 
In the next subsection, the functional elements of an HEV traction component group are defined and parametrized. More precisely, the design of F2M energy transformation element is detailed based on the multi-physical FTG (Fuel Traction component Group).

\section{F2M Transformation Element Design}

The design starts with the definition of input (I) and output (O) power limitations. For a transformation element, energy transfer is bidirectional. Thus, the maximum power of the FTG can be used to define the acceptance of the source port of F2M transformation element (necessary information about source/consumer ports is given in Section 2.2). The acceptance defines the maximum power that the F2M element can receive from another functional element at a given instant. Fig. 9 shows the main components of a FTG.

In order to set the transformation element parameters, the FTG is considered as a black-box component for which I/O ports have to be defined. As the FTG function is to transform fuel energy into mechanical energy, I/O energies are not the same. Thus, the F2M functional element receives fuel energy on the input port (consumer port) and provides a mechanical energy on the output port (source port). Therefore, the input port is connected to the fuel storage element and the delivered mechanical energy is mainly used for vehicle motion (differential and vehicle wheels at multi-physical level) and is partially recovered to recharge the battery.

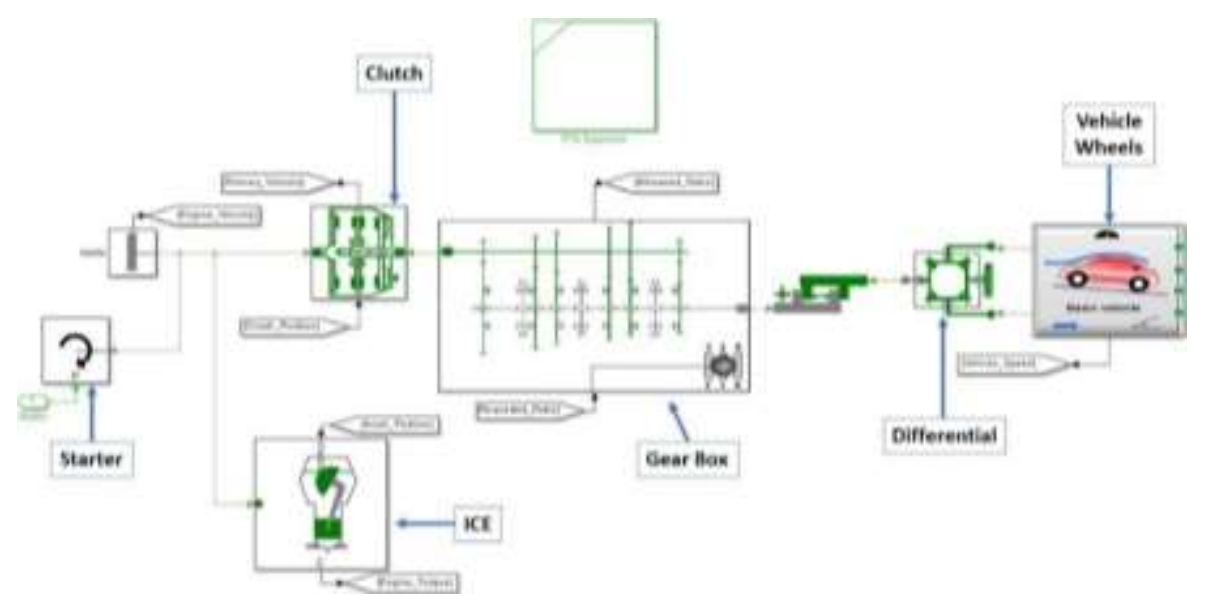

Fig. 9. FTG system of a vehicle.

The acceptances from vehicle to F2M and from F2M to fuel storage are parametrized once the I/O ports are defined. As the ICE cannot have regenerative braking like the electric motor, the acceptance from vehicle to F2M is set to 0 . This means that during deceleration phases the mechanical brakes are activated and F2M is not used as a source of torque anymore. On the other hand, the acceptance from F2M to fuel storage is chosen as the maximum output power of the FTG, including the overall component efficiency. 


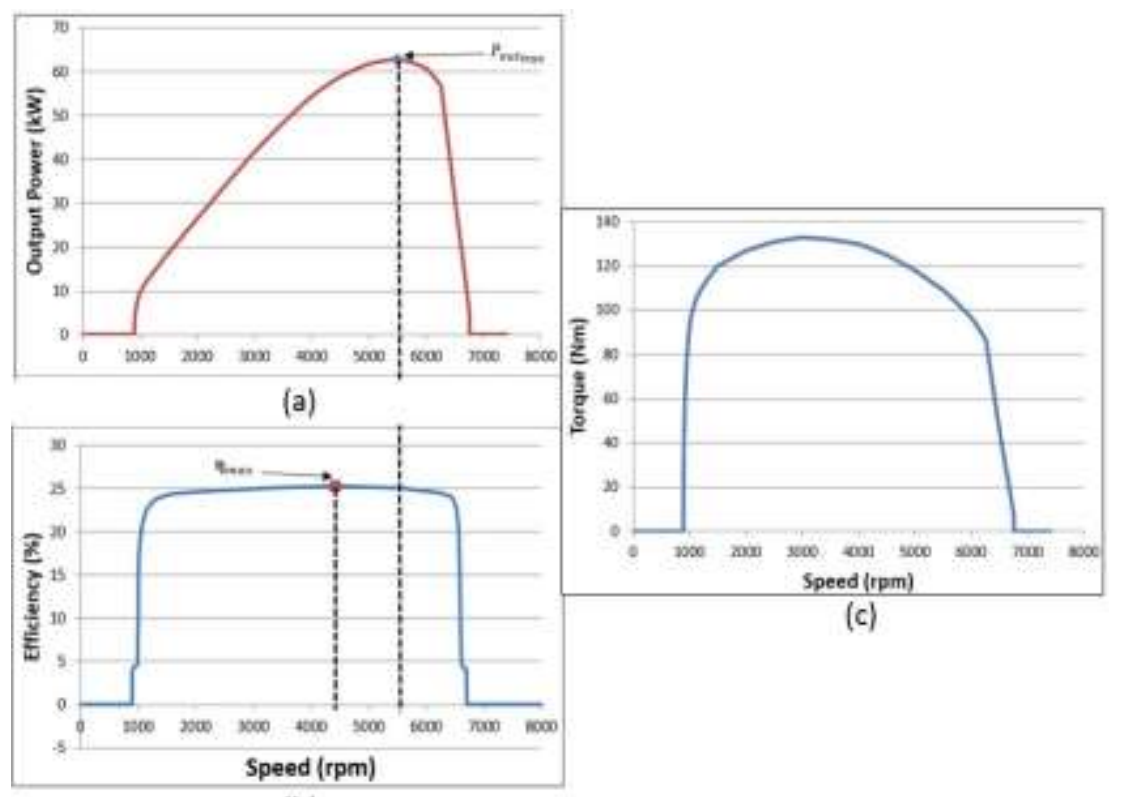

(b)

Fig. 10. ICE static characteristics (a) Output mechanical power of ICE, (b) Efficiency of ICE and (c) Output torque of ICE.

The maximum output power of a FTG can be obtained from the manufacturer. Fig. 10 presents the static characteristics of an ICE. The ICE without gear box has similar characteristics as an electrical machine. If the physical system of FTG regroups the ICE with a gearbox, the characteristics of the engine is not enough to anticipate the maximum output power to the vehicle wheels. As all vehicles with FTG have a gearbox with at least three gear shifts, the efficiency and performance of the FTG change.

An example of maximum output power-speed characteristics of an ICE with its gearbox is given in Fig.11. The static characteristics depend on the gear shifts values that change the FTG performances. From these characteristics, the global maximum output power is derived (represented in Fig. 11 with a dot line). The maximum value of the overall characteristics yields the maximum output power for the F2M transformation element. In this example, the maximum output power of FTG is of approximately $62 \mathrm{~kW}$. This means that for an ICE output power of $70 \mathrm{~kW}$ (manufacturer's value) and a FTG output power (after the gear box and shaft) of $62 \mathrm{~kW}$, the mechanical power losses are about $8 \mathrm{~kW}$.

Still, to determine the acceptance from F2M to fuel storage, the knowledge of the FTG efficiency is required. Torque values can be calculated for a given speed and output power with the following relation:

$$
\tau_{n} \cdot \omega_{n}=P_{\text {out }_{n}}
$$

where $\tau$ is the torque, $n$ is the gear ratio number, $\omega$ is the given angular speed and $P_{\text {out }}$ is the output power of the FTG. 
Torque-speed characteristics for three gear shifts with the associated operating zones are given in Fig. 12 and the efficiency-torque characteristics of FTG is shown in Fig. 13. These values are obtained by modification of gear ratio with manufacturer's torquespeed curve. The local controller of FTG has to keep the system in these operating zones to maximize the efficiency of the component group. The efficiency value of F2M transformation element is determined as the average of maximum efficiencies of all operating zones, which means that the local controller of FTG forces the component group to operate in these zones (see Fig.12).

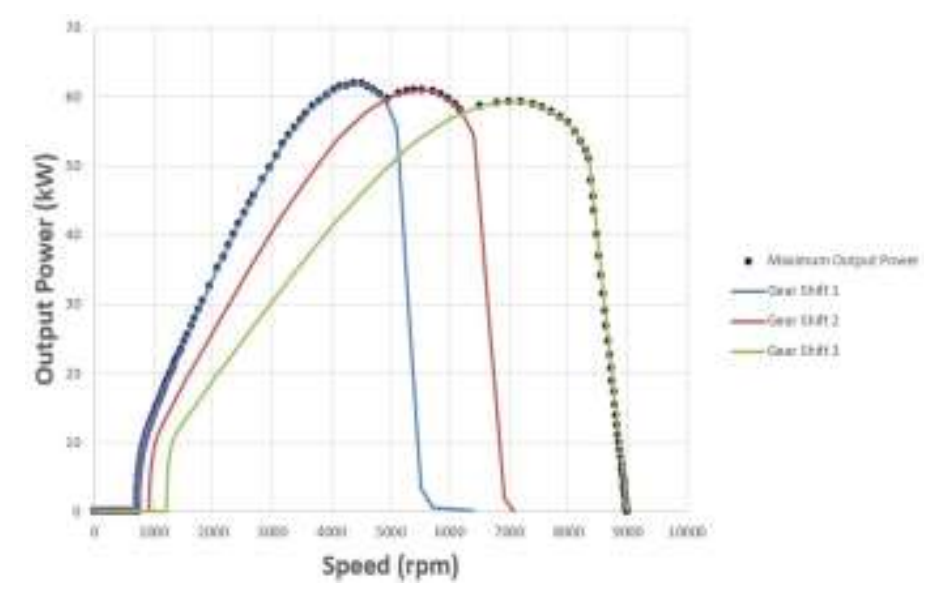

Fig. 11. FTG output power characteristics with three gear shifts.

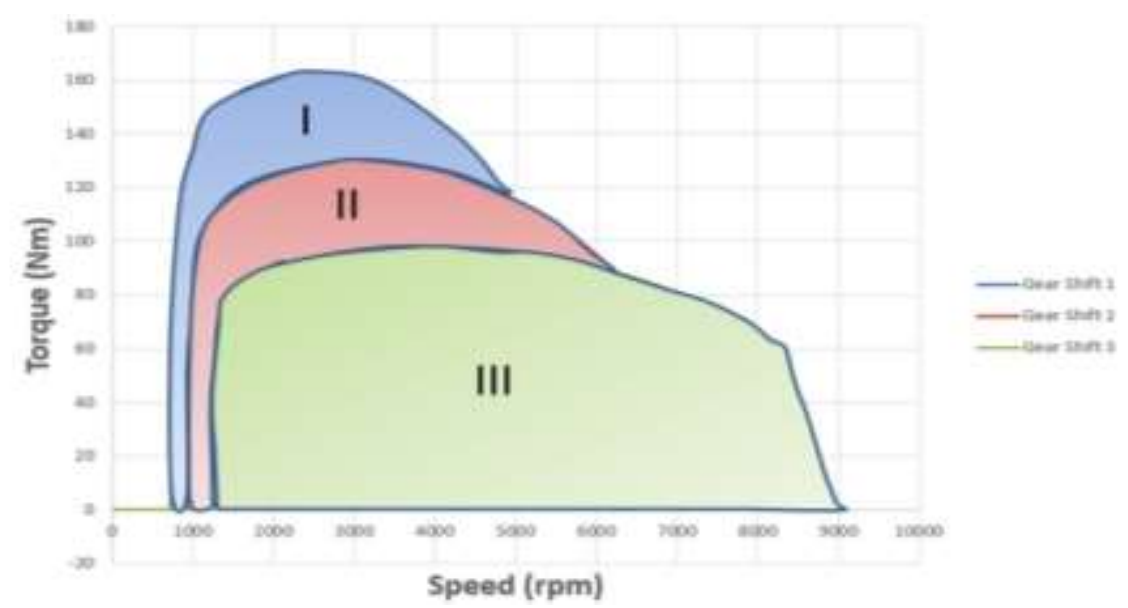

Fig. 12. Torque-speed characteristics for three gear shifts with high efficiency operating zones. 


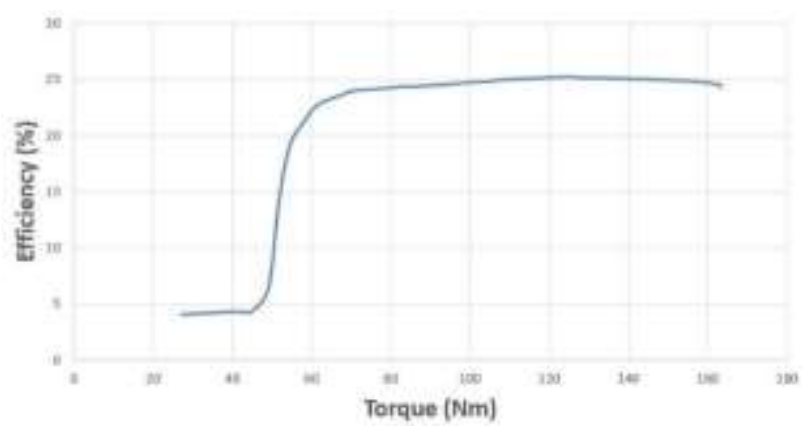

Fig. 13. Efficiency - torque static characteristics.

As the local controller of FTG is considered to be working at maximum efficiency at each point, low efficiency values of low torque operating points are neglected. For this example, the acceptance of F2M is calculated as follows:

$$
P_{a c c_{1}}=P_{o u t_{1}} \cdot \frac{1}{\eta_{1-2}}=70 \mathrm{~kW} \cdot \frac{1}{0.25}=280 \mathrm{~kW}
$$

where $P_{a c c_{1}}$ is the acceptance of input port (consumer port), $P_{\text {out }}$ is the output power of output port (source port) and $\eta_{1-2}$ is the efficiency from consumer port to source port. The parametrization of F2M is summarized in Table 2.

Table 2. Final parametrization of F2M.

\begin{tabular}{|l|c|}
\hline \multicolumn{1}{|c|}{ Parameter } & Value \\
\hline $\begin{array}{l}\text { Efficiency from input port to output } \\
\text { port }\end{array}$ & 0.25 \\
\hline $\begin{array}{l}\text { Efficiency from output port to input } \\
\text { port }\end{array}$ & 0 \\
\hline $\begin{array}{l}\text { Acceptance of F2M element to fuel } \\
\text { storage }\end{array}$ & $280 \mathrm{~kW}$ \\
\hline $\begin{array}{l}\text { Acceptance to F2M element from vehi- } \\
\text { cle }\end{array}$ & 0 \\
\hline
\end{tabular}

Furthermore, the parametrization of the E2M transformation element can be done in a similar way. This functional element represents the function of the Electric Traction Component Group (ETG) that has only one gear ratio. In this case, the global efficiency takes into account the invertor and the gear efficiencies. Yet, the difference is the capacity of the E2M element to absorb regenerative breaking energy, which means that the efficiency and the acceptance from vehicle to E2M element have to be determined.

With the right parametrization of functional model elements, the resource allocation and optimization algorithm are validated and ready to be used for further simulations. Hence, the functional model can be adapted to design the supervision system (GRM) of the multi-physical model. Section 4 details the design of the control architecture at multi-physical level. 


\subsection{Control Architecture}

The functional model of the parallel plug-in hybrid vehicle has been developed in [6]. The developed model, shown in Fig. 14, is used to compute the power split between the multiple sources of the system, for different configurations and missions of the vehicle. Moreover, it allows to evaluate the fuel consumption, maximum speed, maximum acceleration and regenerative braking power [6].

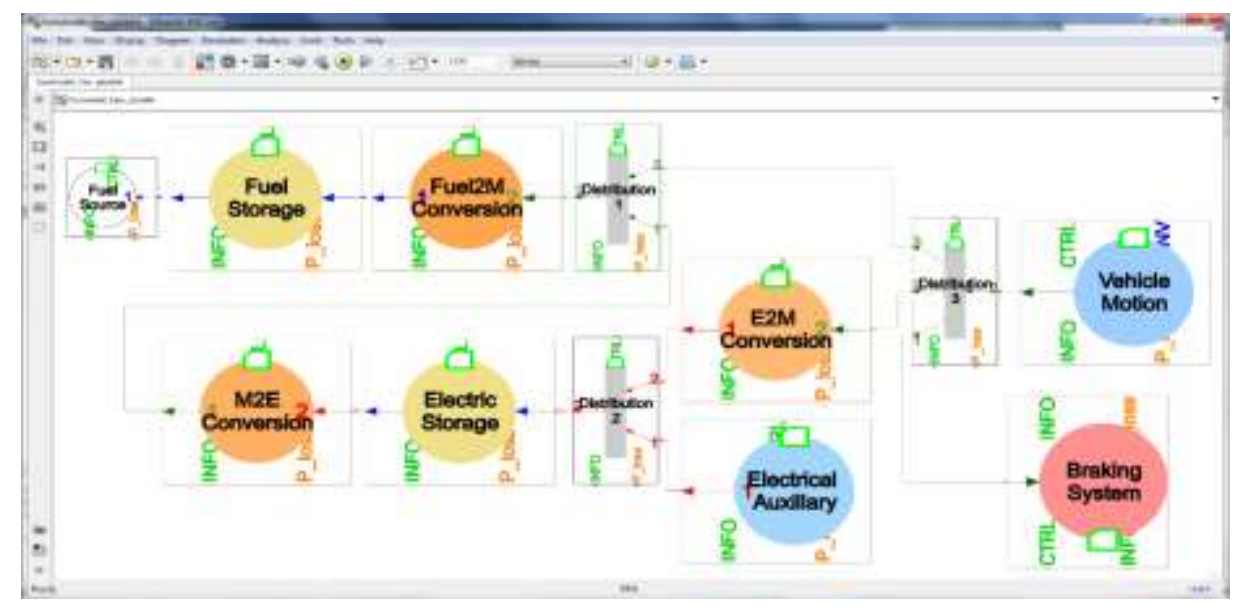

Fig. 14. Functional model of PHEV [6].

Thereafter, the next step in the design process is to use the information provided by the functional model (i.e. power signals for each source) for control design of a more complex multi-physical model. To this purpose, the power signals are transformed into physical reference signals using a unique interconnection element, which is added to each element of the functional model to adjust the flow nature, as shown in Fig. 15.

Using this link, the functional and multi-physical models are able to exchange necessary values of power or physical references, as well as measured/estimated values.

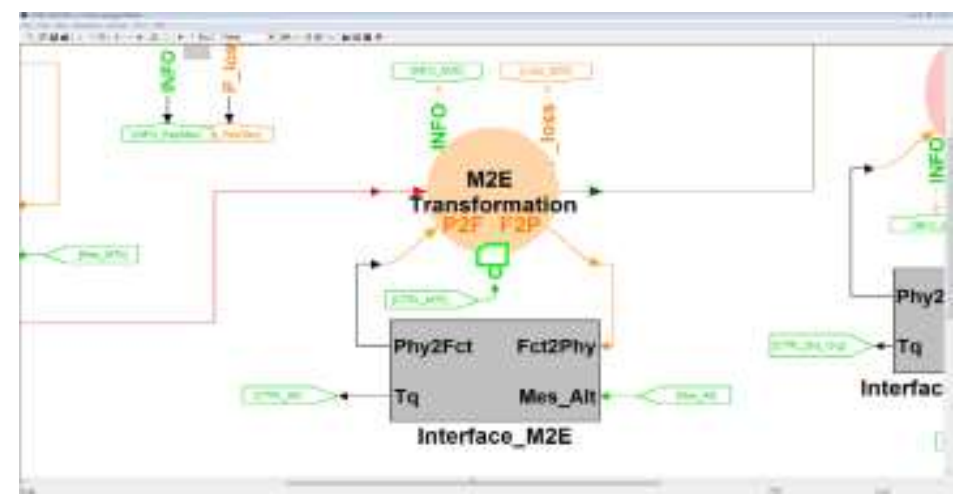

Fig. 15. Functional model of M2E transformation element with its connection element [11] 
The multi-physical model of the system is given in Fig. 16, where each component is a system itself. For example, Fig. 17 illustrates the representation of the electric machine subsystem.

As it can be noticed, the system architectures are similar in both functional and multiphysical models. Thus, if there is any change at multi-physical level, the functional model has to be adapted respectively.

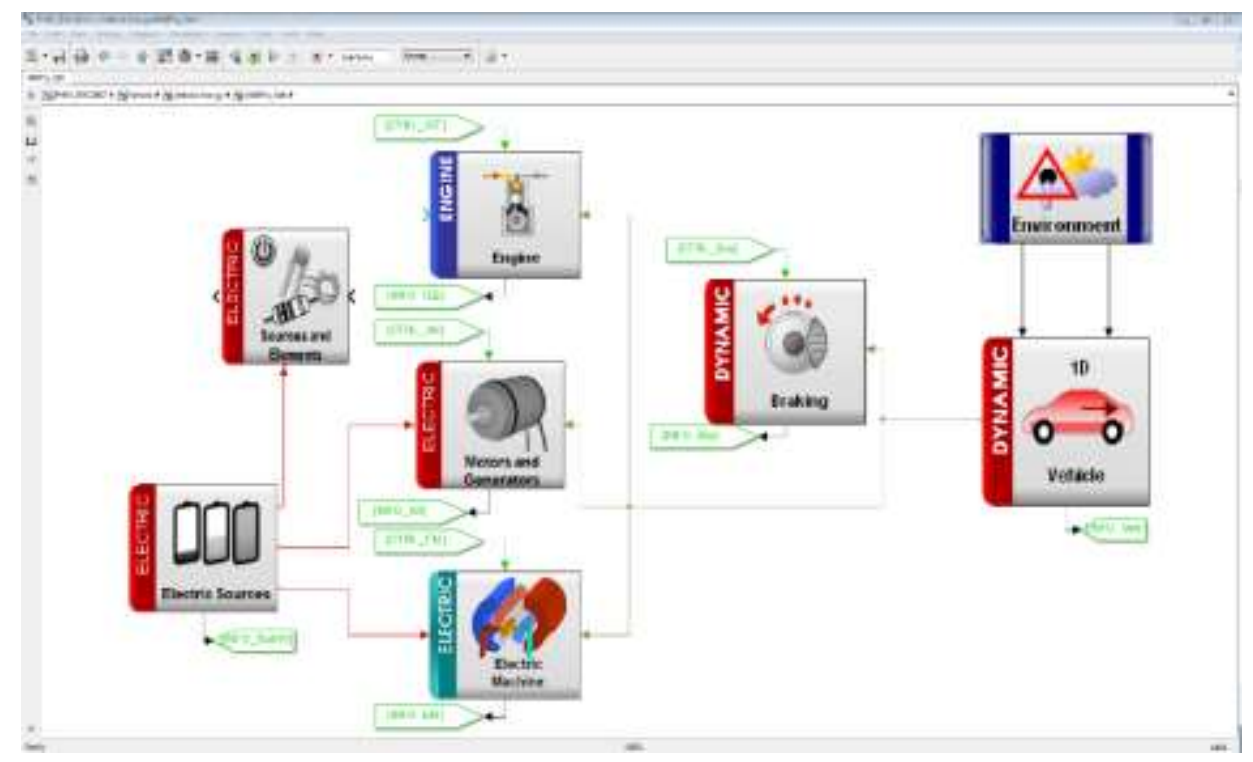

Fig. 16. Multi-physical model of HEV [11].

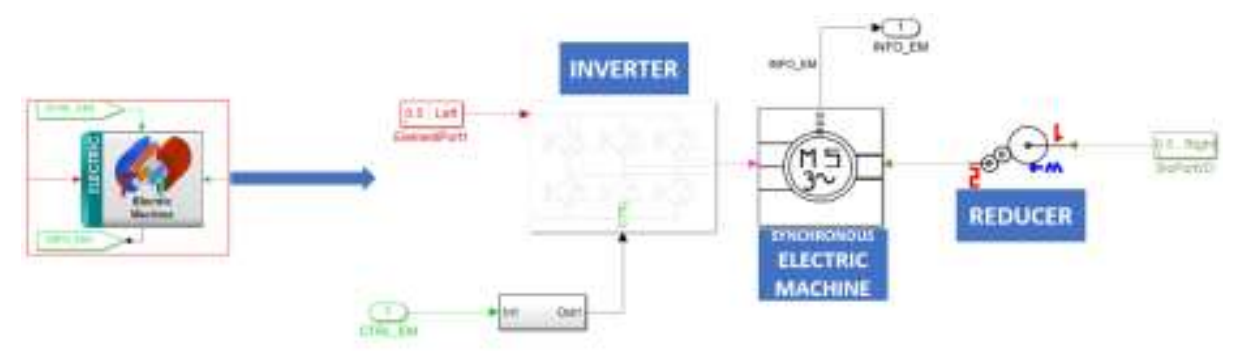

Fig. 17. Electric machine component group [11].

\subsection{Simulation Results}

The simulations are run under the following assumption: the vehicle always moves forward. The model parameters are consistent with those of a PHEV available on the market. Table 3 provides the technical characteristics of the vehicle. 
Table 3. Technical characteristics of PHEV.

\begin{tabular}{|c|c|}
\hline Technical Characteristics & Value \\
\hline Fuel tank max. volume & 451 \\
ICE max. output power & $70 \mathrm{~kW} @ 5000 \mathrm{rpm}$ \\
ICE max. output torque & $140 \mathrm{Nm} @ 4500 \mathrm{rpm}$ \\
\hline Battery voltage & $210 \mathrm{~V}$ \\
Battery capacity & $50 \mathrm{Ah}$ \\
\hline EM max. output power & $60 \mathrm{~kW}$ \\
EM max. output torque & $200 \mathrm{Nm}$ \\
\hline Combined max. output power & $100 \mathrm{~kW}$ \\
\hline Vehicle curb mass & $1500 \mathrm{~kg}$ \\
Vehicle SCx (Aerodynamic drag coeff.) & 0.63 \\
Vehicle wheel radius & $0.635 \mathrm{~m}$ \\
\hline
\end{tabular}

To be able to compare the simulation results with the manufacturers' brochure, the vehicle performance indicators are determined and their values are given in Table 4.

Table 4. Performance indicators of PHEV.

\begin{tabular}{|c|c|}
\hline Performance Data & Value \\
\hline Combined consumption (WLTC) & $3.21 / 100 \mathrm{~km}$ \\
\hline Electric drive range & $25 \mathrm{~km}$ \\
\hline Vehicle max. speed & $180 \mathrm{~km} / \mathrm{h}$ \\
\hline Vehicle max. speed in e-drive mode & $85 \mathrm{~km} / \mathrm{h}$ \\
\hline Vehicle max. acceleration $(0-100 \mathrm{~km} / \mathrm{h})$ & $11.4 \mathrm{~s}$ \\
\hline
\end{tabular}

First of all, the functional model with its DM is simulated using the WLTC (Worldwide harmonized Light Vehicles Test Cycle) that yields the vehicle speed and the power demand illustrated in Fig. 18(a), (b). In addition, the DM uses a ruled-based energy management strategy based on priorities, which is implemented in the distribution element of the functional model. In this example, the functional model has three main distribution elements that are detailed in Table 5 with their priorities.

Table 5. Distribution element priorities.

\begin{tabular}{|c|c|c|c|}
\hline Priority No & Distribution 1 & Distribution 2 & Distribution 3 \\
\hline 1 & Drive & Electrical Aux. & Electric Drive \\
\hline 2 & Battery Charge & Drive & Fuel Drive \\
\hline 3 & N/A & N/A & Brake System \\
\hline
\end{tabular}

Distribution element 1 transmits mechanical energy supply from fuel to mechanical transformation element to drive or mechanical to electrical transformation elements. Distribution element 2 transmits electrical storage energy supply to electrical auxiliary or drive. Distribution element 3 transmits the energy need of vehicle dynamics to electric drive supply element or to fuel drive supply element or to the brake system. 
The obtained results are also shown in Fig. 18. The vehicle speed and power achieve the desired profiles and meet the requirements of the WLTC. The regenerative braking can be observed between 1600s and 1800s in the Fig. 18 (c), (d). The vehicle speed on electric drive is limited to $85 \mathrm{~km} / \mathrm{h}$ and the electrical storage/battery SOC (state of charge) is limited to \%20; beyond these values, the electric drive is abandoned and electrical energy is consumed just by electrical auxiliaries.

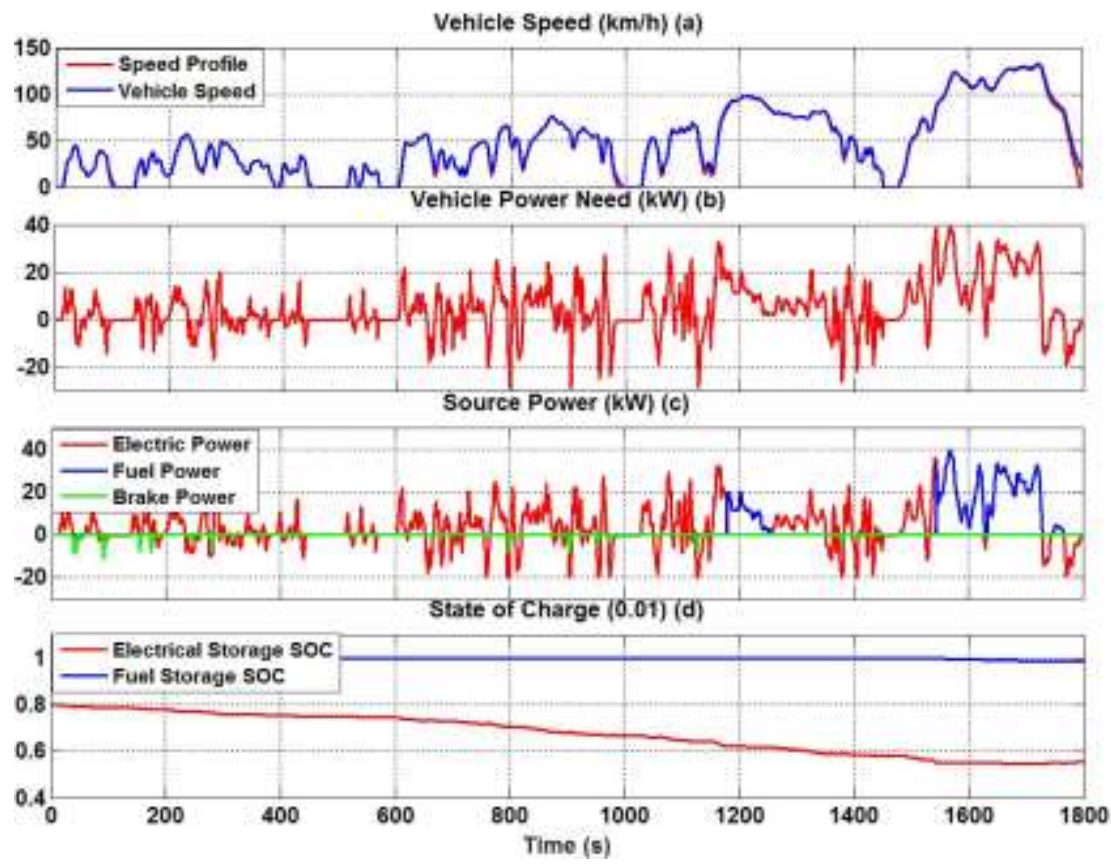

Fig. 18. PHEV - simulation results using the functional model [6].

These results represent a first validation of the chosen architecture of the vehicle and of the energy management strategy used to handle the power split within the system. However, at this level of abstraction, the energy model cannot generate specific physical signals such as electric motor output torque or battery output current.

Simulation results of the multi-physical model and the proposed control architecture are illustrated in Fig. 19. 


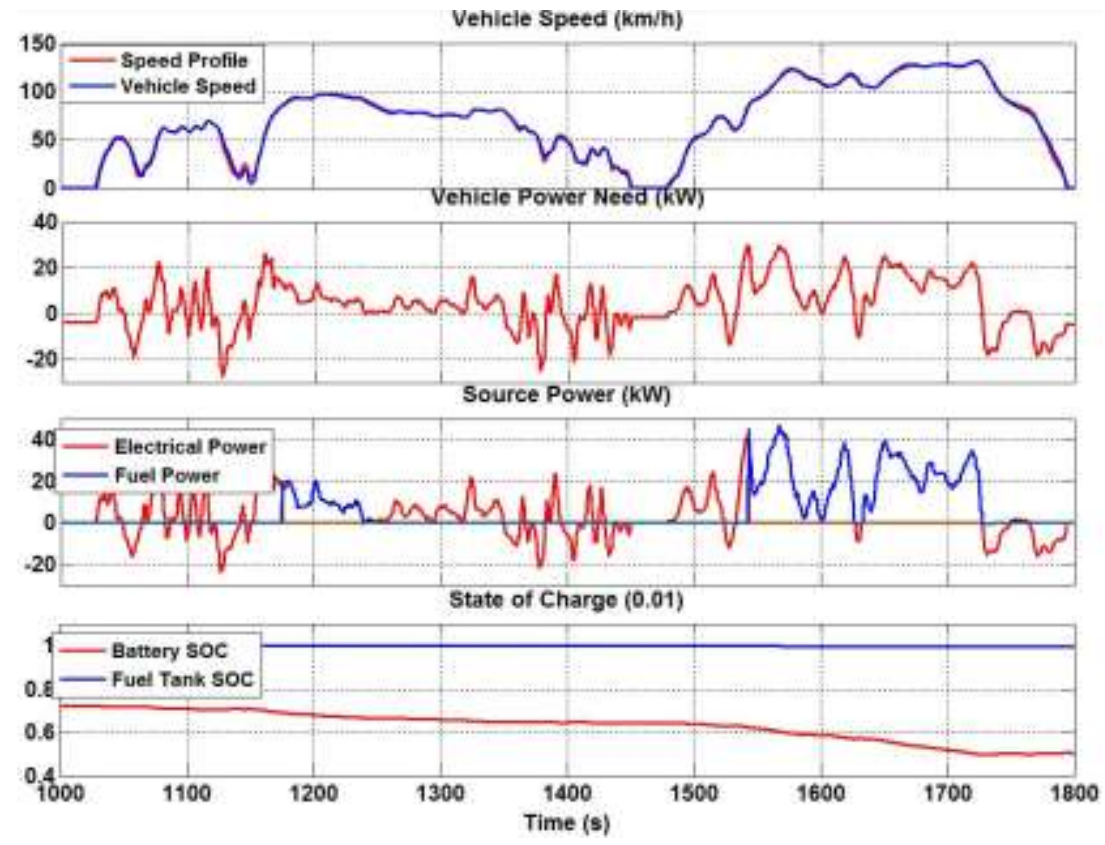

Fig. 19. PHEV - simulation results using the multi-physical model with GRM [11].

According to Fig.19, the following remarks can be made:

- Vehicle power need pattern is compatible with allocated source powers;

- When the vehicle surpasses $85 \mathrm{~km} / \mathrm{h}$ (Fig. 19 (c)) the source power allocation moves to fuel source power, but at the same time battery SOC decreases (Fig. 19 (d)). The reason behind this is the constant electrical auxiliary load;

- A slight increase in battery SOC is seen at the end of the simulation showing the regenerative braking effect;

- Based on the parameters values shown in Table 3, the results are consistent with the physical limits of the components;

- From the acquired data, the fuel consumption can be calculated from following equation:

$$
\frac{\Delta S O C_{\text {fuel }}}{\Delta d} \cdot \operatorname{vol}_{\text {fuel }} \cdot 100
$$

For this test scenario (WLTC), the obtained fuel consumption is of $3.51 / 100 \mathrm{~km}$. This result is well approximated by the value given in Table $4(3.21 / 100 \mathrm{~km})$. Besides the fuel consumption, power need and supply patterns have been compared. Slight differences can be observed due to the system dynamic behavior, especially at time instants with negative power supply.

With the proposed solution, the system can be examined globally but also locally. Each component of the vehicle can be investigated separately if the simulation model permits. Fig. 20 shows the electric machine results. 

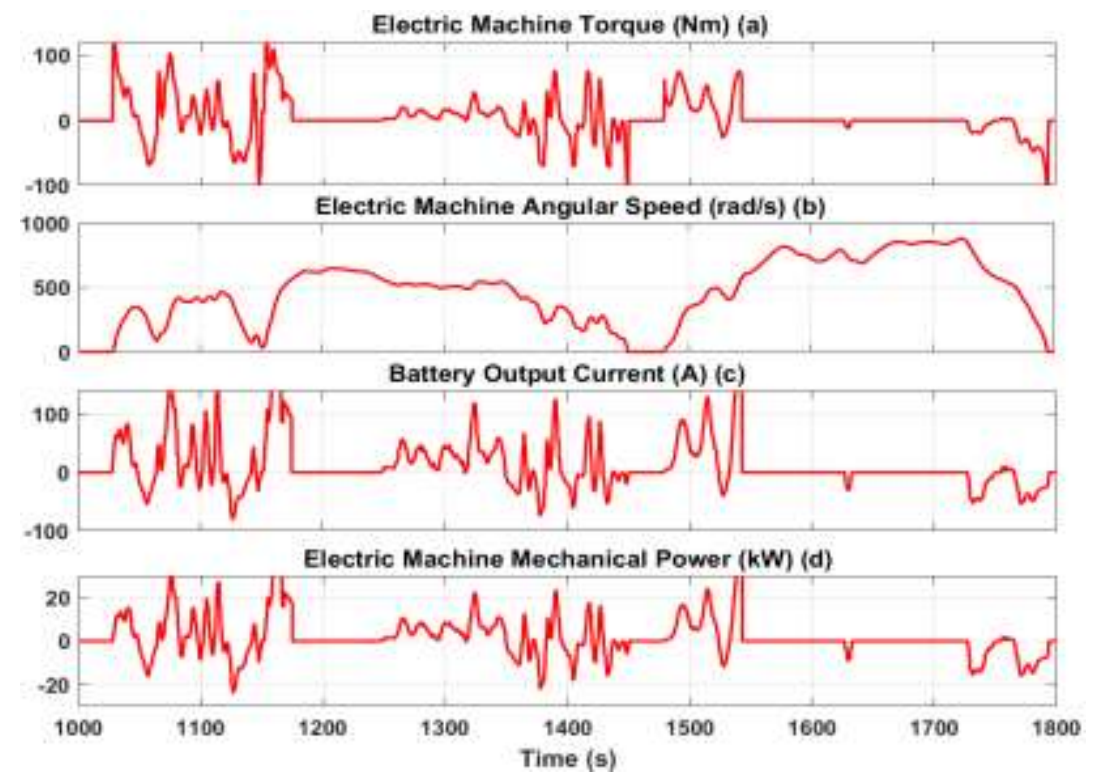

Fig. 20. Hybrid vehicle electric machine results [11].

From the specific physical signals of the electric motor, the following comments can be made using information given in Table.3:

- Output torque values of electric machine are within its physical limits (maximum output torque is $200 \mathrm{Nm}$ );

- The angular speed of the electrical machine follows the vehicle speed with a certain gear ratio;

- The electric motor current is between the physical limits with possibility of detailed analysis for regenerative braking (for example, the motor torque constant $K_{t}$ is approximately 1 , which is acceptable);

- The electric motor mechanical power is illustrated in order to calculate the motor and generator efficiencies.

These results highlight the advantages of a multi-physical model with a GRM: detailed analysis of components, better precision and, therefore, reliable validation of simulations. Other physical components (ICE, auxiliaries, battery etc.) can also be analyzed using the same simulation data. However, data exploitation depends on the multi-physical model complexity.

\subsection{Additional Comments on Reconfiguration}

A major advantage of the functional model is the ability to handle the system architecture reconfiguration without reviewing the analytical modeling, which cannot be avoided for a multi-physical representation of the system. If a component is added or 
removed, the new configuration can be validated in a fast and efficient way. An example of this interesting feature is demonstrated in Fig. 21, highlighting the modularity of functional modelling. Compared to Fig. 14, an additional component is added to the system: a second electrical to mechanical energy conversion element used for traction.

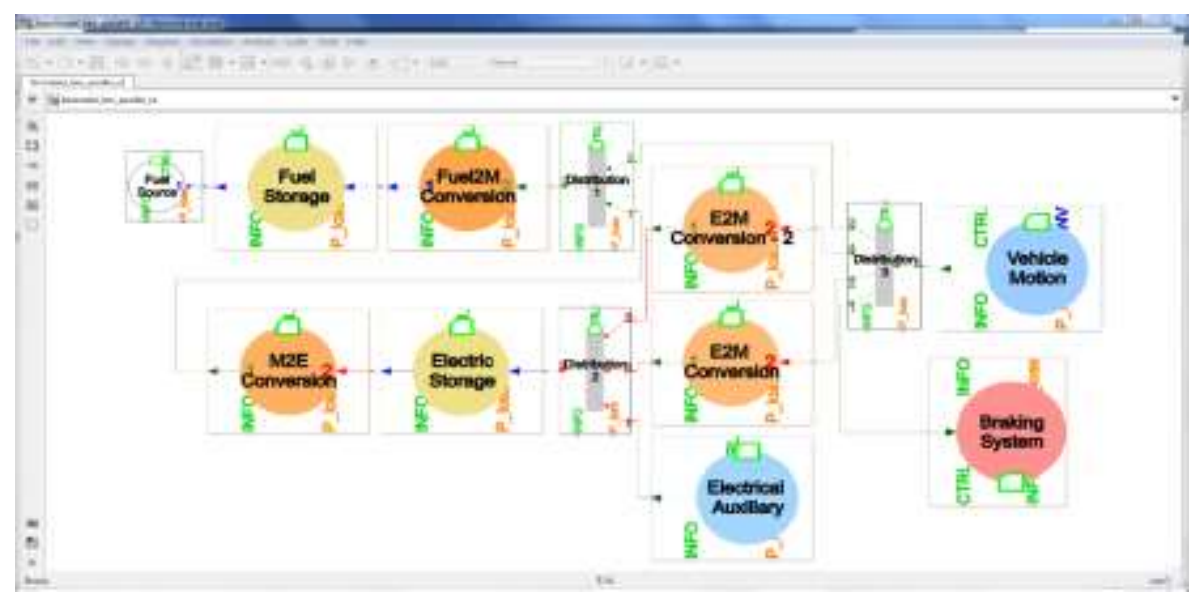

Fig. 21. Reconfigured PHEV model [11].

Therefore, due to faster simulations and ease of reconfiguration, the functional modeling becomes a very useful methodology for system modeling and simulation.

A first validation of the reconfigured PHEV is given in Fig. 22. Here, both E2M transformation elements results are shown, as well as F2M transformation element and all the other components that are used for this simulation.
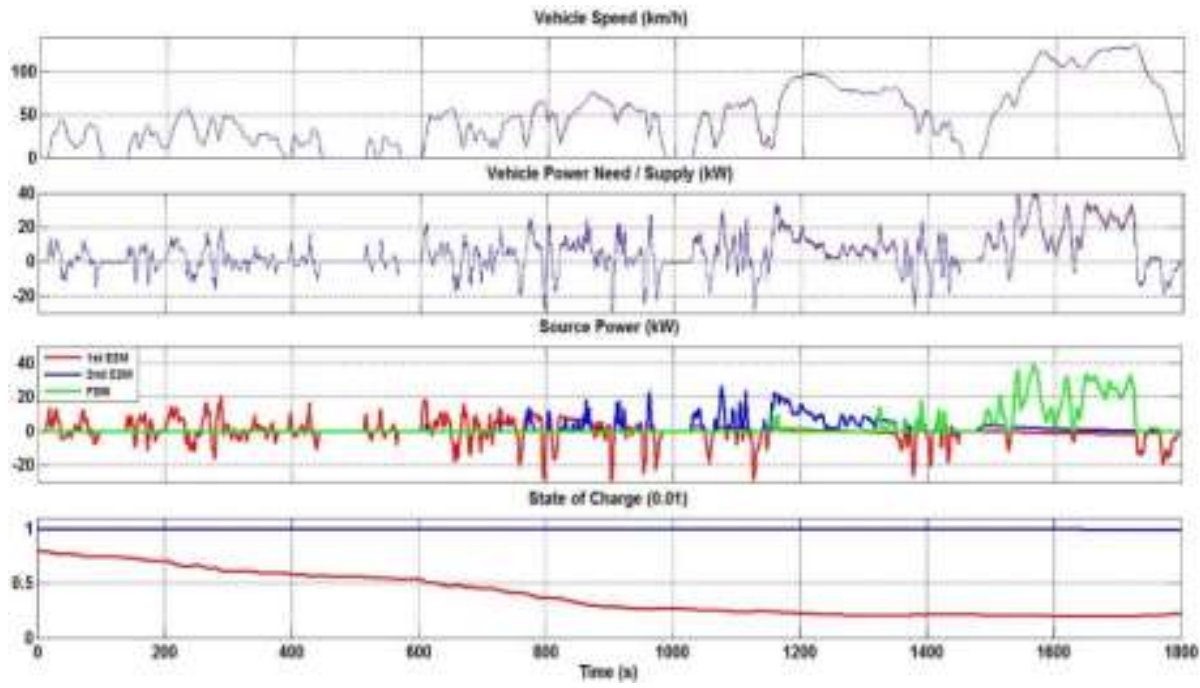

Fig. 22. Simulation results of the reconfigured PHEV model. 
The simulation results of the reconfigured vehicle system show the following advantages of the functional modelling approach:

- Global system efficiency and fuel consumption can be calculated and be compared with values obtained for a vehicle architecture having one E2M conversion element. This type of analysis helps to choose the most appropriate configuration for the system and to perform the elements sizing accordingly.

- Traction and regenerative braking can be handled just by one E2M conversion element or both, and if necessary, F2M conversion element can be used for traction.

After the first validation of system reconfiguration and energy optimization strategy, the obtained power flow information can be transmitted using the proposed solution to the multi-physical model for a more reliable validation.

\section{$5 \quad$ Conclusion and Future Work}

In this study, a methodology for control architecture design is proposed using a functional modelling model. The developed procedure is general and can be applied for complex systems in order to obtain the supervision system for energy flow management.

One of the main advantages of the proposed modelling approach is the possibility to validate the energy distribution strategy, at first, by using an energetic functional model (a reduced complexity model in comparisons with the multi-physical model of the same system). The simplicity of equations and the modularity of the functional model, associated with an appropriate simulation environment, allows to quickly evaluate the energy management strategy using fast simulations, to easily adapt the strategy in case of architecture changes and ultimately to validate the system components sizing.

Another important advantage of this control design methodology is that it uses the system representation at a functional level of abstraction for decision-making at multiphysical level. Therefore, once the functional model is obtained and the interconnection elements are defined, the global resource manager (GRM) is determined and the energy distribution strategy can be evaluated with a more accurate multi-physical model. At this stage, the system performance indices can be computed and compared with the ones from manufacturers' brochures for validation. This procedure simplifies significantly the laborious design process based entirely on the multi-physical representation of a complex system, which makes it of great interest to a large number of companies.

The functional model and the control architecture at multi-physical level are designed and successfully validated for a plug-in hybrid electric vehicle (PHEV). Simulation results are obtained in Matlab/Simulink environment using the WLTC driving cycle. The interconnection between two system representations at different level of abstraction and the functional elements parametrization based on component groups of multi-physical model are detailed for the PHEV system.

Further research on a generalized element of interconnection for two levels of representation will be conducted. 
In addition, the models of multi-physical components can be refined in order to enhance the accuracy of the global multi-physical model (i.e. use more complex models for transformation component groups), which can lead to more relevant/conclusive results.

A long-term perspective is to replace the priority-based energy management algorithm with a more efficient one, based on optimization. The new algorithm has to be integrated within distribution elements such that to ensure an optimum need/supply allocations. For instance, the model predictive control strategy can be considered to optimally manage the power split within the system. Furthermore, the robustness of the control architecture can also be investigated. Finally, the proposed methodology can eventually be applied on different types of applications like water treatment systems or building energy management systems to prove the method's generality.

\section{References}

1. Brunet, J., Flambard, L., Yazman, A.: A hardware in the loop (HIL) model development and implementation methodology and support tools for testing and validation car engine electronic control unit. In: International Conference on Simulation Based Engineering and Studies, TCN CAE, Lecce, Italy (2005).

2. Fauvel, C., Claveau, F., Chevrel, P.: Energy management in multi-consumers multi-sources system: a practical framework. In: IFAC World Congress, pp.2260-2266, Cape Town, South Africa (2014).

3. Fauvel, C. : Approche modulaire de l'optimisation des flux de puissance multi-sources et multi-clients, à visé temps réel. PhD Thesis, Ecole des Mines, Nantes, France (2015).

4. Fiani, P., Ait Taleb, L., Chavanne, S., Mokukcu, M. : Modélisation pour la conception et l'évaluation de systèmes complexes. In: Revue Ingénieurs de l'Automobile. vol.841, SIA, France (2016).

5. Le Moigne, J.L.: La théorie du système général, théorie de la modélisation. PUF, Paris, France (1977).

6. Mokukcu, M., Fiani, P., Chavanne, S., Ait Taleb, L., Vlad, C., Godoy, E., Fauvel, C.: A new concept of functional energetic modelling and simulation. In: The 9th Eurosim Congress on Modelling and Simulation. pp.536-541, Oulu, Finland (2016).

7. Penalva, J.M.: Sagace: la modélisation des systèmes dont la maîtrise est complexe. In: 2nd International Conference on Integrated Logistics and Concurrent Engineering (ILCE). vol.94, pp.536-541, Montpellier, France (1994).

8. Salmasi, F.R.: Control strategies for hybrid electric vehicles: evolution, classification, comparison and future trends. IEEE Transactions on Vehicular Technology(56), 2393-2404 (2007).

9. Sciaretta, A., Guzzella, L.: Control of hybrid electric vehicles. IEEE Control Systems(27), 60-70 (2007).

10. Suh, N.P.: Axiomatic design theory for systems. Journal of Research in Engineering Design(10), 189-209 (1998).

11. Mokukcu, M., Fiani, P., Chavanne, S., Ait Taleb, L., Vlad, C., Godoy, E.: Control Architecture Modeling using Functional Energetic Method: Demonstration on a Hybrid Electric Vehicle. In: Proceedings of the $14^{\text {th }}$ International Conference on Informatics in Control, Automation and Robotics (ICINCO 2017). vol.1, pp.45-53, Madrid, Spain (2017). 